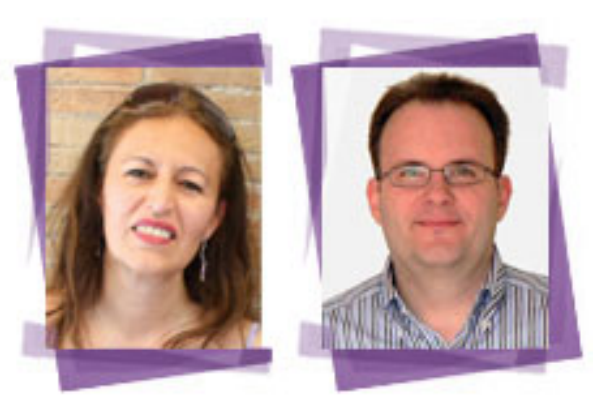

\title{
Localization and web accessibility
}

Emmanuelle Gutiérrez y Restrepo (Fundación Sidar - Acceso Universal)

Loïc Martínez Normand (Facultad de Informática, Universidad

Politécnica de Madrid)

\section{Abstract}

Web content accessibility for people with functional diversity is essential for building and integrating society. Thus, it is important to take into account accessibility when localizing accessible web content. This paper describes the key web content accessibility requirements from the localization viewpoint.

\section{Keywords}

localization, web, web accessibility, accessibility guidelines

\section{Introduction}

Society clearly recognizes equal rights for people with functional diversity (or people with disability), as stipulated in the United Nations' Convention on the Rights of Persons with Disabilities (UN, 2006).

For people with functional diversity to be integrated in today's society, the emphasis has to be placed on access to information and communication technologies (ICT) and, particularly, web content. There are an increasing number of work-related and everyday activities that can be performed on the web, such as shopping, access to public administration services, tax payment, access to media, education, and so on.

In view of this, it is essential that web content is accessible for people with disabilities. The World Wide Web Consortium (W3C) and its Web Accessibility Initiative (WAI) have been working towards this goal since 1997 and have created web accessibility guidelines (W3C, 2010) (WAI, 2010). The most important document in this field is the Web Content Accessibility Guidelines (WCAG), the second version of which was published in December 2008 (Caldwell et al., 2008).

From the viewpoint of the localization of web pages and sites, it is important to maintain the accessibility level of the original content. To do this, the localization process should ensure that the translated version does not fail any of the accessibility requirements that the original version meets. There are several international organizations that are working on internationalization issues in ICT and the web, such as Technical Committee 37 of the International Standards Organization (ISO, 2007), the Localization Industry Standards Association (LISA, 2010) and the W3C Internationalization Activity (W3C, 2010b). But none of these organizations deal specifically with accessibility issues.

With the goal of describing localization-related accessibility issues, this paper presents the WCAG 2.0 requirements that we believe are most relevant from the viewpoint of web content localization. The decision on which requirements are relevant is based on our lengthy experience in working in favour of web accessibility and translating technical documents into Spanish, as part of our work at the Sidar Foundation (Sidar, 2010).

At this point, it is worth mentioning that web accessibility is also beneficial for people without disabilities. For example, a key principle of web accessibility is designing web sites and software that are flexible 
enough to meet different user needs, preferences, and situations. In some circumstances, such flexibility also benefits people without disabilities, such as people using a slow Internet connection, people with "temporary disabilities", such as a broken arm, and people with changing abilities due to aging.

The paper is structured as follows. First, we will give a short overview of WCAG 2.0 to describe its structure and content. Then we will describe the WCAG 2.0 success criteria that are considered to be essential for the localization of accessible web documents. Finally, some conclusions will be drawn.

\section{Overview of WCAG 2.0}

WCAG 2.0 is a W3C recommendation (Caldwell et al., 2008) that was published in December 2008 and contains three main layers of guidance: principles, guidelines and success criteria.

The principles provide the foundation of web accessibility. There are four principles:

- Perceivable: Information and user interface components must be presentable to users in ways they can perceive. It cannot be invisible to all of their senses.

- Operable: User interface components and navigation must be operable. Users must be able to operate the interface, that is, the interface cannot require interaction that a user cannot perform.

- Understandable: Information and the operation of user interface must be understandable. Users must be able to understand the information as well as the operation of the user interface (the content or operation cannot be beyond their understanding).

- Robust: Content must be robust enough that it can be interpreted reliably by a wide variety of user agents, including assistive technologies. Users must be able to access the content as technologies advance (as technologies and user agents evolve, the content should remain accessible).

The second layer of guidance is the set of guidelines. There are twelve guidelines that provide the basic goals that web designers should work toward in order to make content more accessible to people with functional diversity. These guidelines are not testable, but they provide the framework and overall objectives to help web designers to understand the success criteria and better implement the techniques.

The third layer of guidance consists of a set of success criteria. For each guideline, several success criteria are provided. These success criteria are written as testable statements (that is, a person can decide whether some web content conforms to any given success criteria). The success criteria allow WCAG 2.0 to be used where requirements and conformance testing are necessary, such as in design specification, purchasing, regulation and contractual agreements. In order to meet the needs of different groups and different situations, three levels of conformance are defined: A (lowest), AA, and AAA (highest). WCAG 2.0 has a total number of 61 success criteria.

In addition to these three main layers of guidance, an informative document explains how to conform to WCAG 2.0 (Caldwell et al., 2008b) and provides three additional layers of guidance to support web designers when conforming to the success criteria. These additional layers are: sufficient techniques (considered by the W3C to be sufficient to meet the respective success criteria), advisory techniques (additional techniques that can enhance accessibility) and common failures (examples of bad practices).

There is another important section in WCAG 2.0 that has to be taken into account apart from the layers of guidance: the conformance section (W3C, 2008). This section describes five conformance requirements for WCAG 2.0 and how to write conformance claims.

\section{Key WCAG 2.0 success criteria for web localization}


There follows a description of the success criteria that we believe are most important for maintaining the level of accessibility of localized web content. This description will be structured around the four WCAG 2.0 principles: perceptible, operable, understandable and robust.

\subsection{Perceptible}

The key issues for localization under the perceivable principle are related to alternatives for non-text content, captions and audio description for time-based media, sign language, meaningful sequence and background audio.

The first issue is localizing the alternatives of non-text content. Today the web provides an increasing amount of content that is not text-based, such as images, sound, video and interactive controls. These elements are not perceivable for all the users and thus a text alternative has to be provided. This text alternative can then be rendered for the users in the modality that they can perceive, that is, auditory (textto-speech), tactile (Braille) or visual (displayed on the screen) output. When localizing accessible web content it is essential to not only to localize the original content, but also to pay special attention to localizing the alternatives to that content. The success criteria related to alternatives are 1.1.1 Non-text content, 1.2.1 Audio-only and Video-only (Prerecorded), 1.2.8 Media Alternative (Prerecorded), and 1.2.9 Audio-only (Live).

One special case of non-text content is time-based media, which require specific additional accessibility work. First, the audio track is not accessible for deaf and hard-of-hearing people and thus captions should be provided. These captions include the dialogues and additional information about sounds that are essential for understanding the content. Second, the visual track is not accessible for blind and visually impaired people. The solution is to provide an audio description: an auditory explanation of the key content of the visual track. When localizing accessible time-based media, it is essential to also localize both the captions and audio descriptions. The success criteria related to time-based media are 1.2.2 Captions (Prerecorded), 1.2.3 Audio Description or Media Alternative (Prerecorded), 1.2.4 Captions (Live), 1.2.5 Audio Description (Prerecorded) and 1.2.7 Extended Audio Description (Prerecorded).

There are a group of web users that are not comfortable with text-based communication: deaf people whose main language is sign language. These people are not at home with and even find it difficult to understand written text and they prefer to use sign language instead. If a web site uses sign language, then the localization should also cover the content represented in sign language. In this case, localization practitioners should be aware that different countries use different sign languages just as there are many spoken languages. The success criterion related to sign language is 1.2.6 Sign Language (Prerecorded).

An additional perception-related issue is the sequence of the content. If the sequence in which content is presented to the user affects its meaning, then a correct reading sequence should be available to the software that renders this content for the user. When localizing a web page, note that reading sequences are not the same in all languages, implying that special attention should be paid to correctly localizing this reading sequence. The related success criterion is 1.3.2 Meaningful Sequence.

The last issue under the perceivable principle is related to background audio. WCAG 2.0 recommends making sure that the user has access to versions of speech content in which there is either no background audio, or the background sounds can be turned off, or the background sounds are at least 20 decibels lower than the foreground speech. If this type of content is present, localization may add dubbed speech in the target language. If this is done, the dubbed speech should work as the foreground audio, and the other sounds, including the original speech, must comply with the above background constraints. The related success criterion is 1.4.7 Low or No Background Audio.

\subsection{Operable}

The key issues for localization under the operable principle are related to bypassing blocks of content, page titles, focus order, link purpose and headings. 
The first issue in this section is related to groups of links, such as navigation menus. Keyboard users and people who have sequential access to web content (for instance, a speech-based web browser for blind users) have to go through a series of links to reach the main content of a page. Thus it is important to provide a link to skip such navigation elements, and this link is, in some cases, concealed from the users until it obtains keyboard focus. This special link should be located and localized accordingly. The related success criterion is 2.4.1 Bypass Blocks.

In addition, all web pages should be titled. This title is the text that appears in the title bar of the web browser and also appears as the web site name when storing bookmarks. The localization of the page should pay special attention to the page title in order to maintain the information provided by the original version. The related success criterion is 2.4.2 Page Titled.

The third issue is related to focus order, that is, the order in which the components of a web page receive focus when pressing the tab key. WCAG states that the focus order should preserve meaning and operability to ensure that keyboard users have a similar experience to users of pointing devices. From the viewpoint of localization, it is important to bear in mind whether the destination language has a different reading order and/or direction than the original language. If it does, localization practitioners should ensure that the focus order does not change the meaning and operability. The related success criterion is 2.4 .3 Focus Order.

The fourth issue is related to the purpose of links. The idea is that link content (the text and/or image) should clearly indicate where the user will go or what will happen when activating the link. This is useful for people with cognitive impairments and for people (like blind or deaf-blind people) that use link-based browsing: they navigate through the links only rather than viewing the full page. At the lower conformance level, the purpose of the link should be built using the link content and its context. At the higher conformance level, the purpose should be described using the link content only. In any case, localization practitioners should be extremely careful to provide adequate information about the link purpose. The relevant success criteria are 2.4.4 Link Purpose (In Context) and 2.4.9 Link Purpose (Link Only).

The last issue under the operable principle is related to headings, labels and sections. WCAG 2.0 states that headings, labels and section headings should clearly represent their purpose to enable the users to easily navigate through the structure of the document. This is useful for people using the keyboard or for people with cognitive impairments. Thus, web content localization practitioners have to pay special attention to the descriptions contained in headings, labels and sections. The relevant success criteria are 2.4.6 Headings and Labels and 2.4.10 Section Headings.

\subsection{Understandable}

The key issues for localization under the understandable principle are related to language identification, unusual words, reading level, pronunciation, error management and help.

Correctly identifying the human language used in web content is essential for software to correctly handle language changes and present information to the user accordingly. For instance, if the language is correctly identified then text-to-speech technology can use the language to deliver the output and search engines can find pages in the user-defined language. The language has to be determined for both the content of the page and for parts of this content that use a different language (except for proper names). When localizing web content, it is thus essential to correctly indicate both the language of the page and the language of parts, using the codes described in (W3C, 2009). The relevant success criteria are 3.1.1 Language of Page and 3.1.2 Language of Parts.

The second issue is related to unusual words and abbreviations. These can make it difficult for the users to understand the web content and should be defined appropriately. First, unusual words, idioms and jargon should be defined, for example, by providing links to the definition in a separate glossary. Second, 
abbreviations should be provided with mechanisms for identifying their expanded form or meaning. Localization has to maintain the definitions and expansions of the original content and could even add some more if required to enhance the understandability of the localized content. The relevant WCAG 2.0 success criteria are 3.1.3 Unusual Words and 3.1.4 Abbreviations.

The third issue is reading level. WCAG 2.0 specifies that, generally, the text should not require a more advanced reading ability than the lower secondary education level as defined in (UNESCO, 1997). If this is not possible, then the developers should provide an additional version of the text with lower reading ability requirements. This is very important for people that have reading difficulties. During localization, it is essential to maintain the reading level of the original content and to localize the alternative simpler version, if any. The related success criterion is 3.1.5 Reading Level.

The next issue is pronunciation. In some languages the way a word is pronounced affects its meaning. In this case it is helpful for blind and visually impaired people or people with limited reading ability to know what the intended pronunciation of the word is so that these users will be better able to understand the content. This is largely language-dependent, and care should be taken when localizing the web content using several techniques such as the Ruby annotation for Asian languages (W3C, 2001). The related success criterion is 3.1.6 Pronunciation.

The next issue is related to labels, instructions and help related to interactive content, such as forms. This is a key issue for people with comprehension difficulties as well as screen reader users. These users largely benefit from clear and adequate labels of form elements, instructions about form usage and help with the meaning and operation of interactive controls. One key issue is to clearly indicate which form elements are mandatory so that users always know which ones they have to fill in. When localizing these interactive elements, special attention should be paid to making the localized labels, instructions and help useful for the intended users. The relevant WCAG 2.0 success criteria are 3.3.2 Labels or Instructions and 3.3.5 Help.

The last issue in this group is error management. Web content should prevent errors, identify any mistakes made and, finally, make suggestions to help users to correct errors. The aim is to help with data input, especially in the case of sensitive information, such as legal, financial or personal data. During localization the error management-related content should be carefully localized so that it uses the usual terminology and formatting of the target language and region. The relevant success criteria are 3.3.1 Error Identification, 3.3.3 Error Suggestion, 3.3.4 Error Prevention (Legal, Financial, Data) and 3.3.6 Error Prevention (All).

\subsection{Robust}

None of the success criteria under this principle are relevant for localization purposes, as these criteria aim to ensure that the content is machine readable is a consistent way across technologies.

\section{Conclusions}

The localization of web content is a creative and evolving undertaking that can maintain and even increase the accessibility of the original content. And this is really a valuable outcome for the users of the localized content. Also, conformance to accessibility requirements will benefit all users, and not just people with disabilities.

Depending on how much freedom the localization team has to change the original content, structure and presentation, the localized version of a web site could provide more functionality that will benefit its users. In any case, the localized version should never be less accessible than the original version. 
For this reason it is essential for web content localization practitioners to understand and apply the web accessibility guidelines with the goal of providing high quality results that also respect the rights of all users.

\section{References}

Caldwell, B., Cooper, M., Reid, L.G., Vanderheiden, G. (eds.). (2008) Web Content Accessibility Guidelines 2.0. W3C Recommendation. http://www.w3.org/TR/WCAG20/. Date of last update: 2008-12-11. Date of last visit: 2010-07-01.

Caldwell, B., Cooper, M., Reid, L. G., Vanderheiden, G. (eds.) (2008b). Techniques for WCAG 2.0. W3C Working Group Note. http://www.w3.org/TR/WCAG20-TECHS/. Date of last update: 2008-12-11. Date of last visit: 2010-07-01.

LISA (2010). The Localization Industry Standards Association. Erasing borders. Respecting boundaries. http://www.lisa.orgl. Date of last update: 2010-06-28. Date of last visit: 2010-07-01.

ISO (2007). ISO TC 37 In brief - Powerpoint presentation. International Standardization Organization. http://isotc.iso.org/livelink/livelink/fetch/-8864700/8864718/8864727/4705221\%20/ISO TC 37 in brief Powerpoint presentation.pdf?nodeid=4360896\&vernum=-2. Date of last update: 2007-09-24. Date of last visit: 2010-07-01.

Sidar (2010). Accesibilidad en la Red (SIDAR). Fundación Sidar - Acceso Universal (in Spanish). http://www.sidar.org/index.php. Date of last update: 2010-04-08. Date of last visit: 2010-07-01.

UN (2006). Convention on the Rights of Persons with Disabilities and Optional Protocol. United Nations.

UNESCO (1997). International Standard Classification of Education.

http://www.unesco.org/education/information/nfsunesco/doc/isced 1997.htm. Date of last update: 199711-01. Date of last visit: 2010-07-01.

W3C (2001). Ruby Annotation. W3C Recommendation. http://www.w3.org/TR/rubyl. Date of last update: 2001-05-31. Date of last visit: 2010-07-01.

W3C (2008). Understanding Conformance. http://www.w3.org/TR/UNDERSTANDING-

WCAG20/conformance.html. Date of last update: 2008-12-11. Date of last visit: 2010-07-01.

W3C (2009). Language tags in HTML and XML. World Wide Web Consortium.

http://www.w3.org/International/articles/language-tags/Overview.en.php. Date of last update: 2009-09-10. Date of last visit: 2010-07-01.

W3C (2010). World Wide Web Consortium. http://www.w3.org/. Date of last update: 2010-06-22. Date of last visit: 2010-07-01.

W3C (2010b). W3C Internationalization (i18n) activity. World Wide Web Consortium.

http://www.w3.org/International/. Date of last update: 2010-06-23. Date of last visit: 2010-07-01.

WAI (2010). Web Accessibility Initiative. http://www.w3.org/WAI/. Date of last update: 2010-06-17. Date of last visit: 2010-07-01. 